\title{
Inhibitory effect of miR-140-5p on doxorubicin resistance of hepatocellular carcinoma
}

\author{
XIAOJIE GAO $^{1 *}$, YAN JIANG ${ }^{2 *}$ and YINGYING LI ${ }^{1}$ \\ ${ }^{1}$ Department of Pharmacy, The Second Affiliated Hospital of Zhejiang Chinese Medical University, \\ Hangzhou Zhejiang 310005; ${ }^{2}$ Department of Pharmacy, Affiliated Hangzhou First People's Hospital, \\ Zhejiang University School of Medicine, Hangzhou, Zhejiang 310006, P.R. China
}

Received January 31, 2020; Accepted January 5, 2021

DOI: $10.3892 /$ etm.2021.9938

\begin{abstract}
To investigate the role of microRNA (miR)-140-5p in doxorubicin (DOX) sensitivity in hepatocellular carcinoma, miR-140-5p and peptidyl-prolyl cis-trans isomerase NIMA-interacting 1 (PIN1) expression was first evaluated in hepatocellular carcinoma tissues using starBase. Next, in vitro experiments were performed. Cell line expression of miR-140-5p and PIN1 expression was detected by reverse transcription polymerase chain reaction. Cell viability and proliferation were determined by the Cell Counting Kit- 8 and EdU assays. The relationship between miR-140-5p and PIN1 was evaluated by TargetScan and a luciferase reporter system. Western blotting was used to detect the expression of PIN1. It was observed that miR-140-5p was downregulated in hepatocellular carcinoma tissues and cell lines compared with normal samples in HCC or normal liver cells. Gain-offunction experiments revealed that miR-140-5p mimics were able to enhance DOX sensitivity of hepatocellular carcinoma cells. Further studies revealed that PIN1 was a target gene of miR-140-5p. Suppression of PIN1 led to higher DOX sensitivity in hepatocellular carcinoma cells. Finally, when comparing a PIN1-siRNA alone group and a PIN1-siRNA plus miR-140-5p inhibitor group, there was no significant difference in cell viability. Furthermore, miR-140-5p mimics did not reduce the sensitivity of PIN1mut plasmid to DOX in HUH7 and SNU449 cells. The present study demonstrated that miR-140-5p could enhance DOX sensitivity in hepatocellular carcinoma cells by targeting PIN1.
\end{abstract}

Correspondence to: Dr Yingying Li, Department of Pharmacy, The Second Affiliated Hospital of Zhejiang Chinese Medical University, 318 Chaowang Road, Hangzhou, Zhejiang 310005, P.R. China

E-mail: 514912312@qq.com

*Contributed equally

Key words: microRNA-140-5p, doxorubicin, peptidyl-prolyl cis-trans isomerase NIMA-interacting 1, hepatocellular carcinoma

\section{Introduction}

Hepatocellular carcinoma (HCC) is one of the most common malignant tumors worldwide and it is ranked as the sixth most common cancer $(1,2)$. It is the third leading cause of cancer mortality globally, and on average, 781,631 patients succumb to HCC every year (3). The current treatment for this disease is orthotopic liver transplantation and surgical resection. However, although liver transplantation can achieve satisfactory results, due to a lack in donor livers, this treatment option is greatly limited (4). Surgical resection is a common cure. However, a range of patients are initially diagnosed at the advanced stage of the disease and few patients are suitable to undergo surgical resection (5). Chemotherapy is often considered as a primary treatment for patients with advanced HCC to protect them against disease $(6,7)$. However, chemotherapy is effective in only a small proportion of patients with advanced HCC, due to chemoresistance (8). Therefore, overcoming drug resistance and sensitization has recently become a focus of research.

miRNAs are a class of small non-coding RNAs of 18-25 nucleotides that regulate gene expression at both the transcriptional and post-transcriptional levels. miRNAs function as either tumor activators or suppressors by mainly binding to the 3' untranslated region (3'-UTR) of their target mRNA $(9,10)$. It has been reported that multiple miRNAs are involved in drug resistance in cancers $(11,12)$. Ma et al $(13)$ revealed that miR205-5p downregulation decreased gemcitabine sensitivity of breast cancer cells via ERp29 upregulation. miR-182 contributed to cell adhesion-mediated drug resistance in multiple myeloma by targeting PDCD4 (14). A previous study revealed that miR140-5p was significantly decreased in HCC tissues compared to adjacent non-tumorous liver tissues and it suppressed tumor growth and metastasis, thus, becoming a valuable biomarker for HCC prognosis (15). On this basis, it was speculated that miR-140-5p may not only be related to the progression of HCC disease, but also participate in the development of drug resistance. Therefore, the present study aimed to investigate the function of miR-140-5p in regulating DOX resistance in HCC cells.

\section{Materials and methods}

Cell culture. The normal liver cell line (THLE-2) and HCC cell lines (HUH7, SNU387 and SNU449) were purchased 
from the Chinese Academy of Science Cell Bank. All cell lines were cultured in Dulbecco's modified Eagle's medium (DMEM) supplemented with 10\% fetal bovine serum (FBS; both from Gibco; Thermo Fisher Scientific, Inc.) in an incubator containing $5 \% \mathrm{CO}_{2}$ at $37^{\circ} \mathrm{C}$.

$R N A$ extraction and reverse transcription-quantitative polymerase chain reaction $(R T-q P C R)$. Total RNA was extracted using the TRIzol reagent (Thermo Fisher Scientific, Inc.). For PIN1, $1 \mu \mathrm{g}$ RNA was reverse transcribed to cDNA by a PrimeScript RT kit (Takara Biotechnology Co., Ltd.) according to the manufacturer's instructions. The resulting cDNAs were quantified via quantitative PCR using an Applied Biosystems 7500 Fast Real-Time PCR system (Thermo Fisher Scientific, Inc.) with a SYBR Premix Ex Taq II kit (Takara Biotechnology Co., Ltd.) according to the manufacturer's instructions. The conditions of PCR were as follows: $94^{\circ} \mathrm{C}$ for $20 \mathrm{sec}$ followed by 40 cycles of $95^{\circ} \mathrm{C}$ for $30 \mathrm{sec}, 60^{\circ} \mathrm{C}$ for $34 \mathrm{sec}$ and $72^{\circ} \mathrm{C}$ for $30 \mathrm{sec}$. The relative quantification was performed using the comparative $2^{-\triangle \Delta C q}$ method (16). The specific primers for PIN1 and miR-140-5p detection are were as follows: PIN1 forward, 5'-TTTGAAGACGCCTCGTTTGC-3' and reverse, 5'-GTGCGGAGGATGATGTGGAT-3'; miR-140-5p forward, 5'- ACACTCCAGCTGGGCAGTGGTTTTACCCTA-3' and reverse, 5'-TGGTGTCGTGGAGTCG-3'; GAPDH forward 5'-CGGAGTCAACGGATTTGGTCGTAT-3' and reverse 5'-AGCCTTCTCCATGGTGGTGAAGAC-3'; U6 forward 5'-GCTTCGGCAGCACATATACTAAAAT-3' reverse 5'-CGCTTCACGAATTTGCGTGTCAT-3'. For miR-140-5p, $1 \mu \mathrm{g}$ RNA was used for target specific reverse transcription (TaqMan MicroRNA Reverse Transcription kit; Applied Biosystems; Thermo Fisher Scientific, Inc.) according to the manufacturer's instructions with the same conditions as above.

Western blotting. Cells were lysed in ice-cold RIPA buffer (Beyotime Institute of Biotechnology) and quantified using a BCA Protein Assay Kit (Beyotime Institute of Biotechnology). Protein $(20 \mu \mathrm{g})$ was separated by $10 \%$ SDS-PAGE and transferred to polyvinylidene difluoride membranes. The membranes were blocked with 5\% non-fat dry milk in $1 \mathrm{X}$ TBST buffer at $37^{\circ} \mathrm{C}$ for $1 \mathrm{~h}$ and incubated with the following primary antibodies (all, Cell Signaling Technology, Inc.; 1:1,000) overnight at $4^{\circ} \mathrm{C}$ : EZH2 (cat. no. 4905), E-cadherin (cat. no. 3195) and Vimentin (cat. no. 5741). Samples were subsequently washed with $1 \mathrm{X}$ TBST, and then incubated with HRP-conjugated secondary antibodies (cat. no. 7074; 1:2,000; Cell Signaling Technology, Inc.) for $2 \mathrm{~h}$ at room temperature. The membranes were imaged using chemiluminescence (EMD Millipore).

Cell viability assay. Cell viability was detected using Cell Counting Kit-8 (CCK-8; Dojindo Molecular Technologies, Inc.) according to the manufacturer's protocol. Briefly, HUH7 or SNU449 cells $(5,000$ cells/well) were seeded in 96-well plates and cultured for $24 \mathrm{~h}$. After treating the cells with various concentrations of DOX $(0,0.0525,0.15,0.3,0.6$ and $1.2 \mu \mathrm{g} / \mathrm{ml})$ for $48 \mathrm{~h}$, CCK-8 solution $(10 \mu \mathrm{l})$ was added into each well and incubated for 2-4 $\mathrm{h}$ in an incubator with $5 \% \mathrm{CO}_{2}$ at $37^{\circ} \mathrm{C}$. The absorbance at $450 \mathrm{~nm}$ of each well was detected using a microplate reader (Bio-Rad Laboratories, Inc.).
Transfection assay. miR-140-5p mimics (5 nM), miR140-5p inhibitor (5 $\mathrm{nM})$, miR-negative control mimics (NC mimics; $5 \mathrm{nM}$ ), miR-negative control inhibitor (NC-inhibitor; $5 \mathrm{nM}$ ), NC small interfering RNA (NC siRNA; $20 \mu \mathrm{M}$ ) and PIN1 siRNA1 were obtained from Shanghai Gemma Biotech. The siRNA sequences targeting PIN1 $(20 \mu \mathrm{M})$, miR-140-5p mimics, inhibitor, negative control (NC) mimics and NC inhibitor were as follows: PIN1 siRNA1, 5'-CCGUGUUCACGGAUUCCGGCA UCCA-3' and PIN1 siRNA2, 5'-GCCCUGGAGCUG AUCAACGGCUAC A-3'; miR-140-5p mimic, 5'-CAGU GGUUUUACCCUAUGGUAG-3'; or NC mimic, 5'-CUCAC CAAAAACCCUAUGGUAG-3'; miR-140-5p inhibitor, 5'-CUACCAUAGGGUAAAACCACUG-3'; or NC inhibitor, 5'-UCUACUCUUUCUAGGAGGUUGUGA -3'. The transfection was performed using Lipofectamine ${ }^{\circledR} 2000$ (Invitrogen; Thermo Fisher Scientific, Inc.) according to the manufacturer's protocol, the transfection medium was replaced with complete medium $6 \mathrm{~h}$ after transfection at $37^{\circ} \mathrm{C}$, after which the cells were incubated for the indicated times. All treatments were started at $24 \mathrm{~h}$ after transfection.

Cell proliferation analysis. Cell proliferation was determined using a Click-iTEdU Imaging kit (Invitrogen; Thermo Fisher Scientific, Inc.) according to the manufacturer's protocol. HCC cells were incubated with the IC50 concentration of DOX (SNU449, $1.313 \mu \mathrm{g} / \mathrm{ml} ; \mathrm{HUH} 7,0.3534 \mu \mathrm{g} /$ $\mathrm{ml}$ ) for $24 \mathrm{~h}$ at $37^{\circ} \mathrm{C}$, followed by $10 \mu \mathrm{M}$ EdU for $2 \mathrm{~h}$ prior to fixation (4\% paraformaldehyde at room temperature for $20 \mathrm{~min})$, permeabilization $(0.5 \%$ Triton-X100 for permeabilization at room temperature for 15-20 $\mathrm{min}$ ), and EdU staining at room temperature for $20 \mathrm{~min}$. Cell nuclei were stained with Hoechst 33342 (Invitrogen; Thermo Fisher Scientific, Inc.) at a concentration of $5 \mu \mathrm{g} / \mathrm{ml}$ for $30 \mathrm{~min}$ at room temperature.

Dual luciferase assay. 293T cells (Type Collection of the Chinese Academy of Sciences) were seeded in 24-well plates and the cells were co-transfected with $5 \mathrm{ml} \mathrm{miR-140-5p \text {or }}$ control at a concentration of $10 \mathrm{nM}$ and $100 \mathrm{ng}$ of wild-type or 3'-UTR mutant (mut) of the PIN1 firefly luciferase reporter plasmid (Promega Corporation) using Lipofectamine 2000 (Invitrogen; Thermo Fisher Scientific, Inc.) according to the manufacturer's protocol. After incubation for $48 \mathrm{~h}$, firefly and Renilla luciferase activities were measured by Dual-Glo ${ }^{\circledR}$ Luciferase reporter assay (cat. no. E2920; Promega Corporation).

Statistical analysis. All data were calculated and analyzed using GraphPad Prism 5.0 (GraphPad Software, Inc.) and presented as the mean \pm standard deviation (SD). StarBase (http://starbase.sysu.edu.cn/index.php) was used to analyze the expression of miRNA in HCC tissues and normal tissue (16). TargetScan (http://www.targetscan.org/vert_71/) predicted the potential target genes of miRNA. Pearson correlation analysis determined the correlation between miR-140-5p and PIN1 expression. The differences between two groups were compared with an unpaired Student's t-test. Results were considered statistically significant at $\mathrm{P}<0.05$. All experiments were repeated three times. 
A hsa-miR-140-5p with 370 cancer and 50 normal samples in LIHC

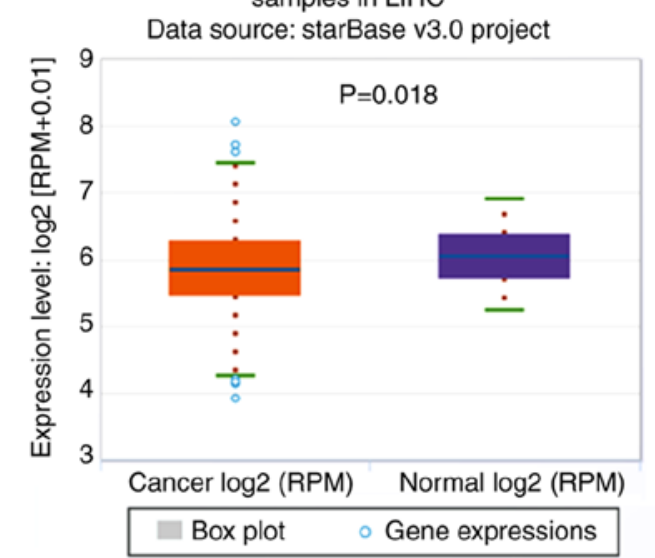

B

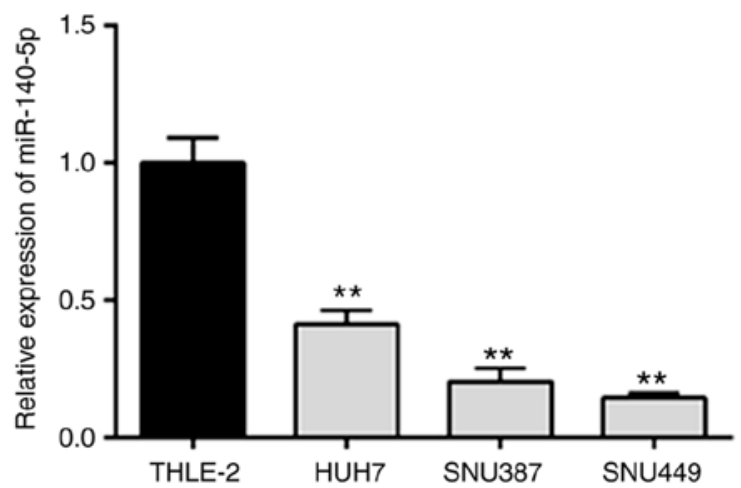

C
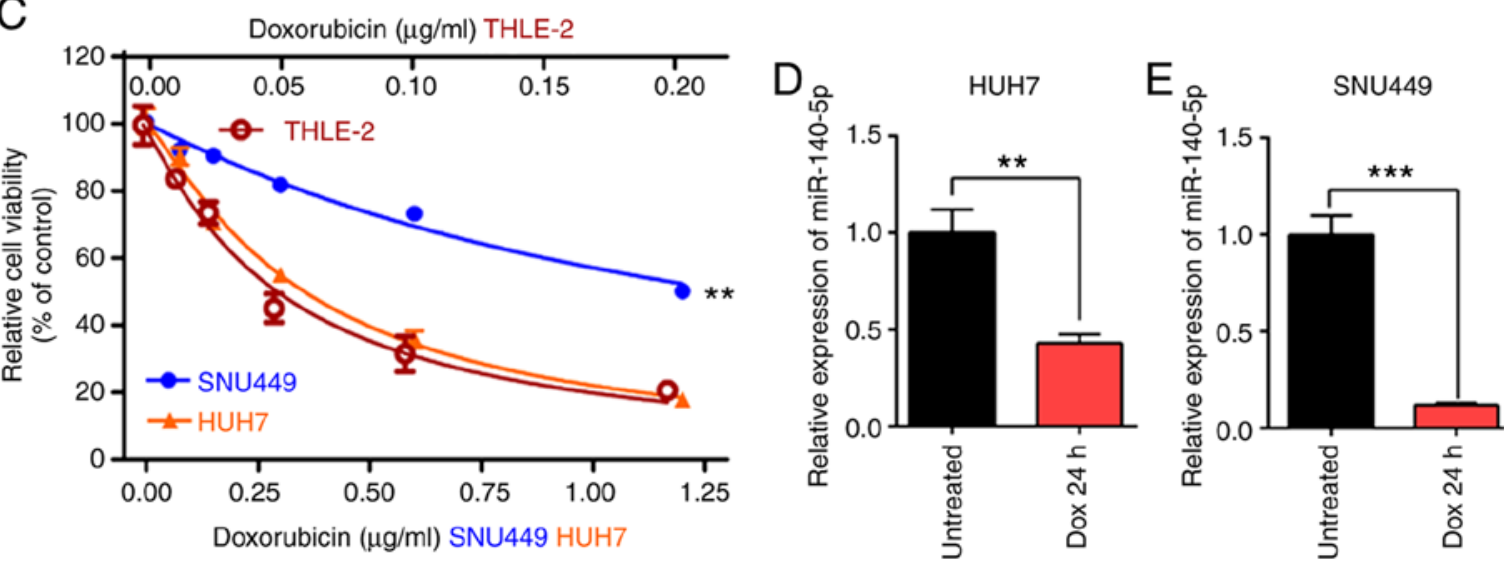

Figure 1. Expression of miR-140-5p in the HCC samples and cell lines. (A) Expression level of miR-140-5p from starBase v3.0 database. (B) Endogenous expression levels of miR-140-5p were detected in normal liver cells (THLE-2) and the HCC cell lines (HUH7, SNU387 and SNU449) using RT-qPCR. ${ }^{* *} \mathrm{P}<0.01$, compared to THLE- 2 cells. (C) The sensitivity of HUH7 and SNU449 cells to DOX. ${ }^{* *} \mathrm{P}<0.01$. (D and E) The expression of miR-140-5p was detected in HUH7 and SNU449 cells exposed to DOX for $24 \mathrm{~h}$. ${ }^{* *} \mathrm{P}<0.01$ and ${ }^{* * *} \mathrm{P}<0.001$ compared to the untreated group. miR-140-5p, microRNA-140-5p; HCC, hepatocellular carcinoma; DOX, doxorubicin; RT-qPCR, reverse transcription-quantitative polymerase chain reaction.

\section{Results}

Expression of miR-140-5p is downregulated in HCC. miR140-5p expression was first analyzed in HCC tissues using starBase (based on 370 cancer samples and 50 normal samples) and it was revealed that the expression level of miR-140-5p was significantly lower in HCC tissues than in adjacent normal tissues ( $\mathrm{P}=0.018)$ (Fig. 1A). Next, miR-140-5p expression was analyzed in a normal liver cell line (THLE-2) and HCC cell lines (HUH7, SNU387, SNU449) and it was revealed that the expression level of miR-140-5p was significantly lower in HCC cell lines compared with the THLE-2 cell line (Fig. 1B). In addition, the expression of miR-140-5p in the epithelial cells (HUH7) was higher than that in the mesenchymal cells (SNU387 and SNU449), and thus, HUH7 and SNU449 cells were selected for the following experiment. These results revealed that miR-140-5p acted as a suppressor gene in HCC.

Next, HUH7 and SNU449 cells were treated with DOX for $24 \mathrm{~h}$ and then CCK-8 and RT-qPCR assays were performed to detect the cellular cytotoxicity and the expression level of miR140-5p. The results revealed that SNU449 was more resistant to DOX when compared with HUH7 (Fig. 1C). Moreover, DOX treatment could reduce miR-140-5p expression both in HUH7 and SNU449 cells (Fig. 1D and E).
miR-140-5p mimic enhances the sensitivity of HCC cells to $D O X$. To investigate the function of miR-140-5p in HCC cell sensitivity to DOX, miR-140-5p mimics, miR-140-5p inhibitor, $\mathrm{NC}$ mimics or NC inhibitor were transiently transfected into HUH7 and SNU449 cells. The efficiency of transfection was confirmed by RT-qPCR assay (Fig. 2A). The results of the CCK-8 assays revealed that the overexpression of miR-140-5p markedly sensitized HUH7 or SNU449 cells to DOX, while miR-140-5p inhibitor markedly reduced sensitivity to DOX compared with the cells transfected with NC inhibitor (Fig. 2B and C). Moreover, it was revealed that the miR-140-5p mimic caused the EdU-positive ratio of HUH7 and SNU449 cells to be significantly decreased upon DOX exposure, compared with the NC mimic group (Fig. 2D and E). These data indicated that miR-140-5p was able to increase DOX sensitivity in HCC cells.

PIN1 is a direct target of miR-140-5p. To determine whether the PIN1 gene was the direct target of miR-140-5p, TargetScan was used to predict the potential target gene of miR-140-5p. It was revealed that PIN1 may be a target of miR-140-5p (Fig. 3A and B). To confirm the association between miR-140-5p and the 3'-UTR of PIN1, a luciferase reporter plasmid containing the 3'-UTR of PIN1 was used. Luciferase activity assays 

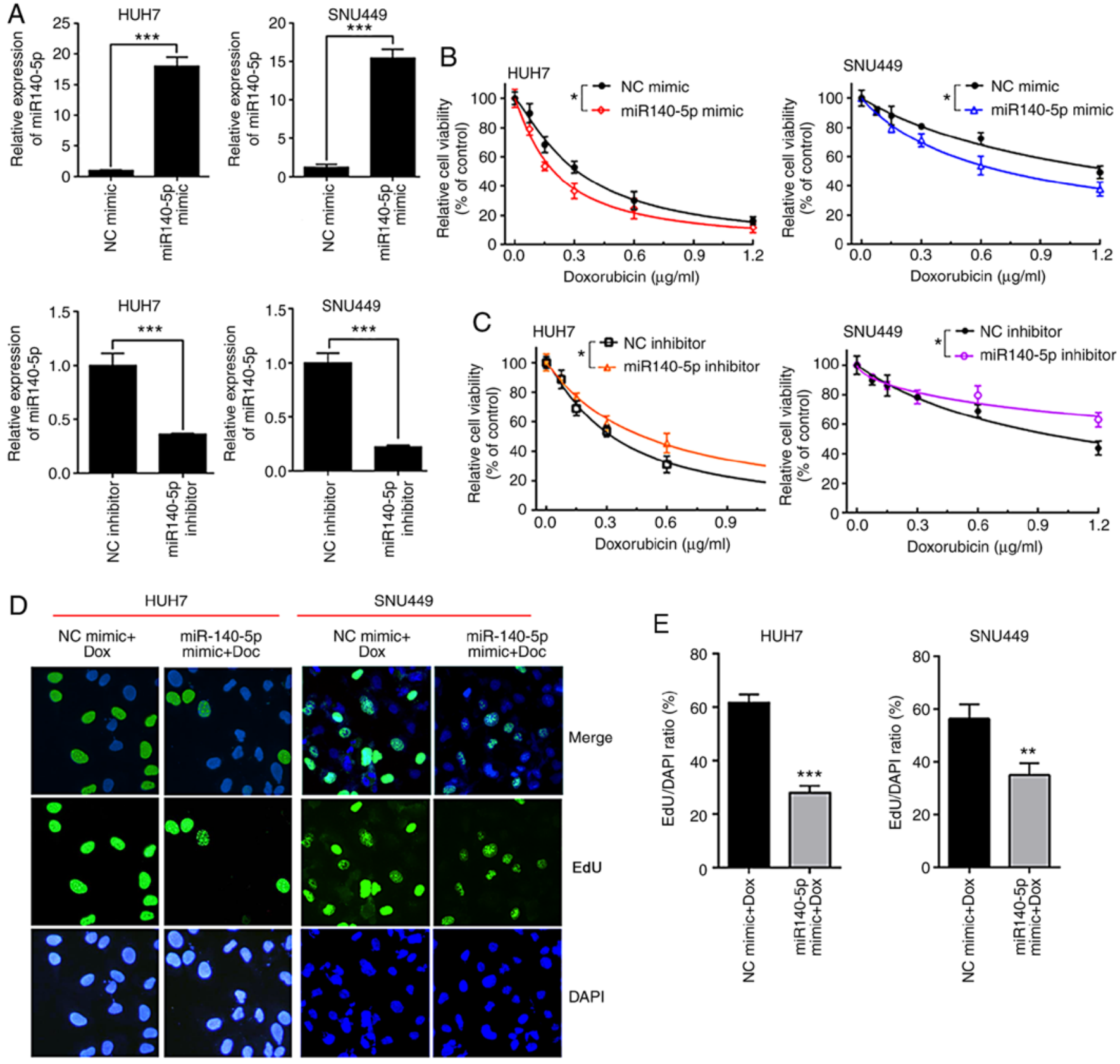

Figure 2. miR-140-5p mimic enhances the sensitivity of HCC cells to DOX. (A) miR-140-5p was detected by RT-qPCR in HUH7 and SNU449 cells treated with miR-140-5p mimics or NC mimic, miR-140-5p inhibitor or NC inhibitor. ${ }^{* * * *} \mathrm{P}<0.001$. (B and C) Cells were treated with different concentrations of DOX for $48 \mathrm{~h}$, and then cell viability was detected using a CCK-8 assay. Overexpression of miR-140-5p sensitized HUH7 cells and SNU449 cells to DOX; inhibition of miR-140-5p (miR-140-5p inhibitor) enhanced resistance to DOX in HUH7 cells and SNU449 cells. "P<0.05. (D and E) Cell proliferation was measured using the EdU assay and the EdU-positive cell ratio was calculated in cells transfected with miR-140-5p mimics or NC mimics. The data in all experiments are presented as the mean $\pm \mathrm{SD}$ of three independent experiments. ${ }^{* *} \mathrm{P}<0.01$ and ${ }^{* * *} \mathrm{P}<0.001$ compared to the NC mimic + Dox. miR-140-5p, microRNA-140-5p; HCC, hepatocellular carcinoma; DOX, doxorubicin; RT-qPCR, reverse transcription-quantitative polymerase chain reaction; NC, negative control; CCK-8, Cell Counting Kit-8; SD, standard deviation.

revealed that miR-140-5p mimics decreased the luciferase activity of the wild-type PIN1 3'-UTR, and this inhibition was offset by the mutation of the target sequences in the PIN1 3'-UTR (Fig. 3C). Next, it was verified whether miR-140-5p downregulates PIN1 in HUH7, SNU449, THLE-2 and SNU387 cells. When these cells were transfected with the miR-140-5p mimic, the expression levels of PIN1 mRNA and protein were significantly suppressed compared to cells transfected with $\mathrm{NC}$ mimics (Fig. 3D and E). These results indicated that PIN1 is a direct target of miR-140-5p.
Knockdown of PIN1 enhances the sensitivity of HCC cells to DOX. PIN1 expression was analyzed in HCC tissues using starBase (based on 371 cancer samples and 50 normal samples) and it was revealed that the expression level of PIN1 was significantly higher in $\mathrm{HCC}$ tissues than in adjacent normal tissues $\left(\mathrm{P}<1 \times 10^{-12}\right.$; Fig. 4A). It was also revealed that DOX treatment increased the mRNA level of PIN1 in HUH7 and SNU449 cells (Fig. 4B). Pearson correlation analysis revealed a negative correlation between the expression level of PIN1 and miR-140-5p (Fig. 4C). Next, the role of PIN1 in cell viability 


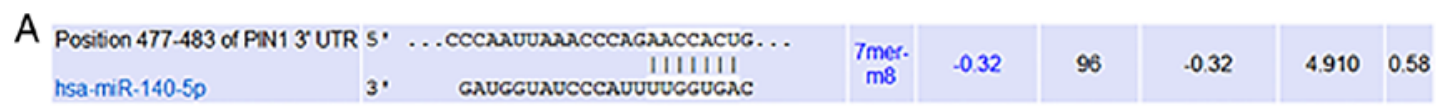

B

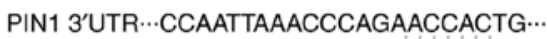
Hsa-miR-140-5p GAUGGUAUCCCAUUUUGGUGAC PIN1 3'UTR-mut ‥CCAATTAAACCCAGTTGGTGAG ‥

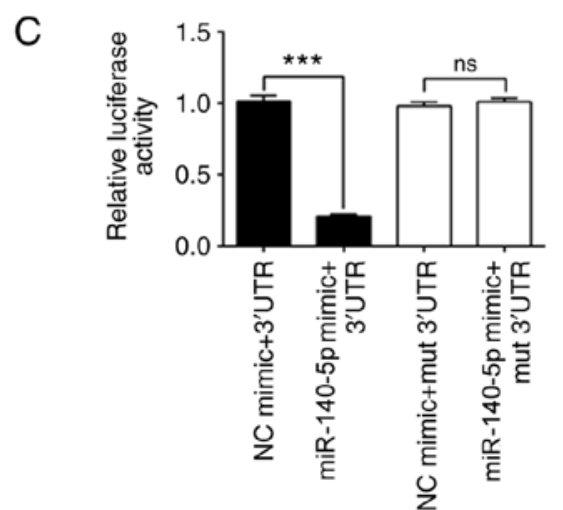

D
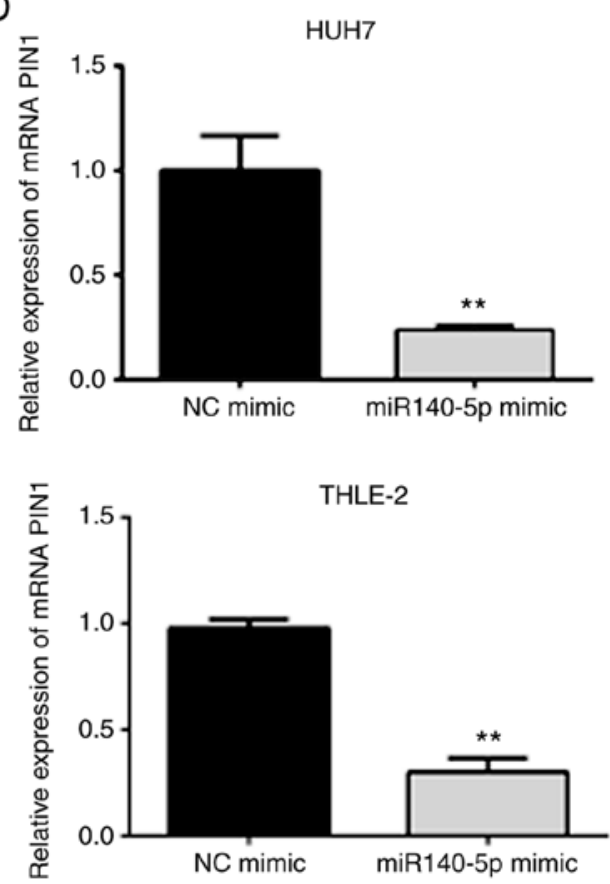

E

$\mathrm{HUH} 7$

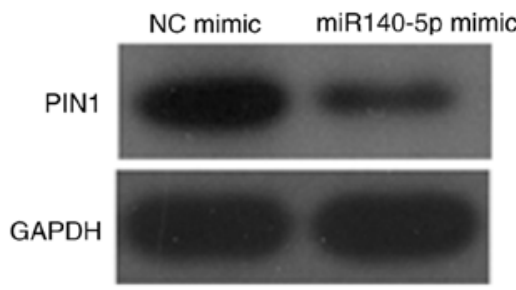

THLE-2

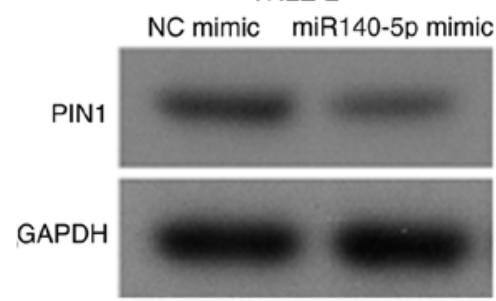

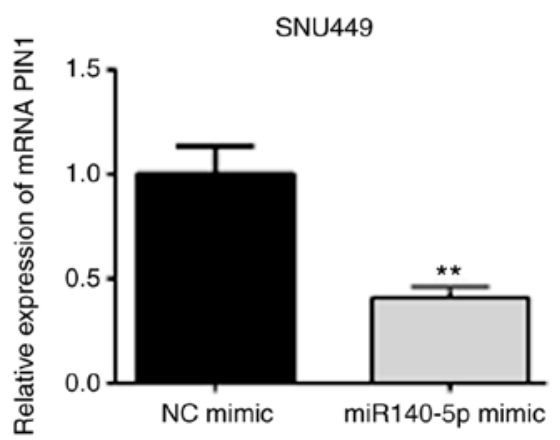

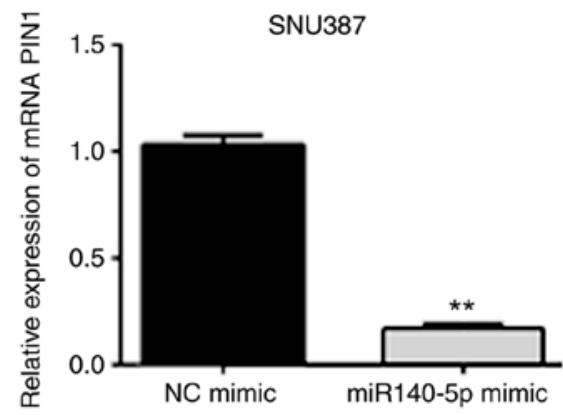

SNU449

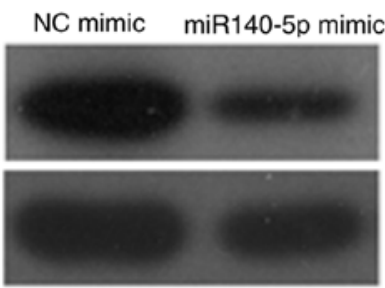

SNU387

NC mimic miR140-5p mimic
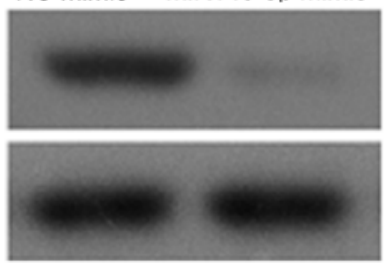

Figure 3. PIN1 is a direct target of miR-140-5p. (A) miR-140-5p was predicted to bind to the 3'-UTR of PIN1 mRNA by TargetScan. (B) Alignment of miR-140-5p with PIN1 3'-UTR. (C) Luciferase assay for the direct targeting of the 3'-UTR of PIN1 by miR-140-5p. ${ }^{* * *} \mathrm{P}<0.001$. (D) PIN1 mRNA expression in HUH7, SNU449, THLE-2 and SNU387 cells transfected with miR-140-5p mimics or NC mimics was analyzed by RT-qPCR. ${ }^{* *} \mathrm{P}<0.01$. (E) PIN1 protein expression in HUH7, SNU449 THLE-2 and SNU387 cells transfected with miR-140-5p mimics or NC mimics was analyzed by western blotting. PIN1, peptidyl-prolyl cis-trans isomerase NIMA-interacting 1; miR-140-5p, microRNA-140-5p; NC, negative control; RT-qPCR, reverse transcription-quantitative polymerase chain reaction. 

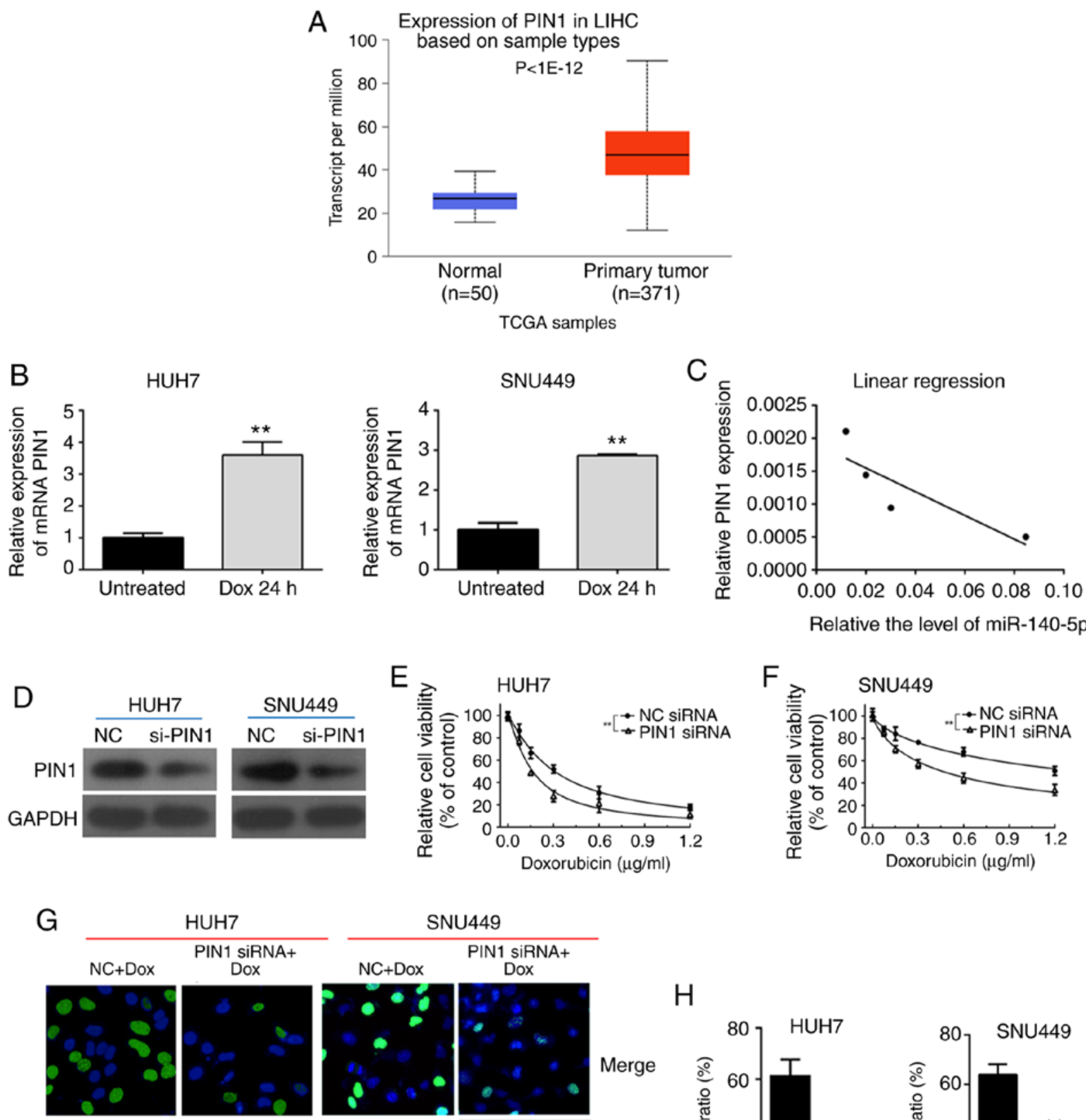

$\mathrm{HUH} 7$

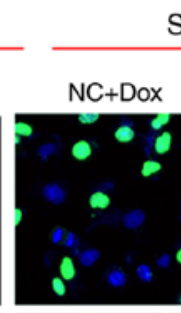

SNU449
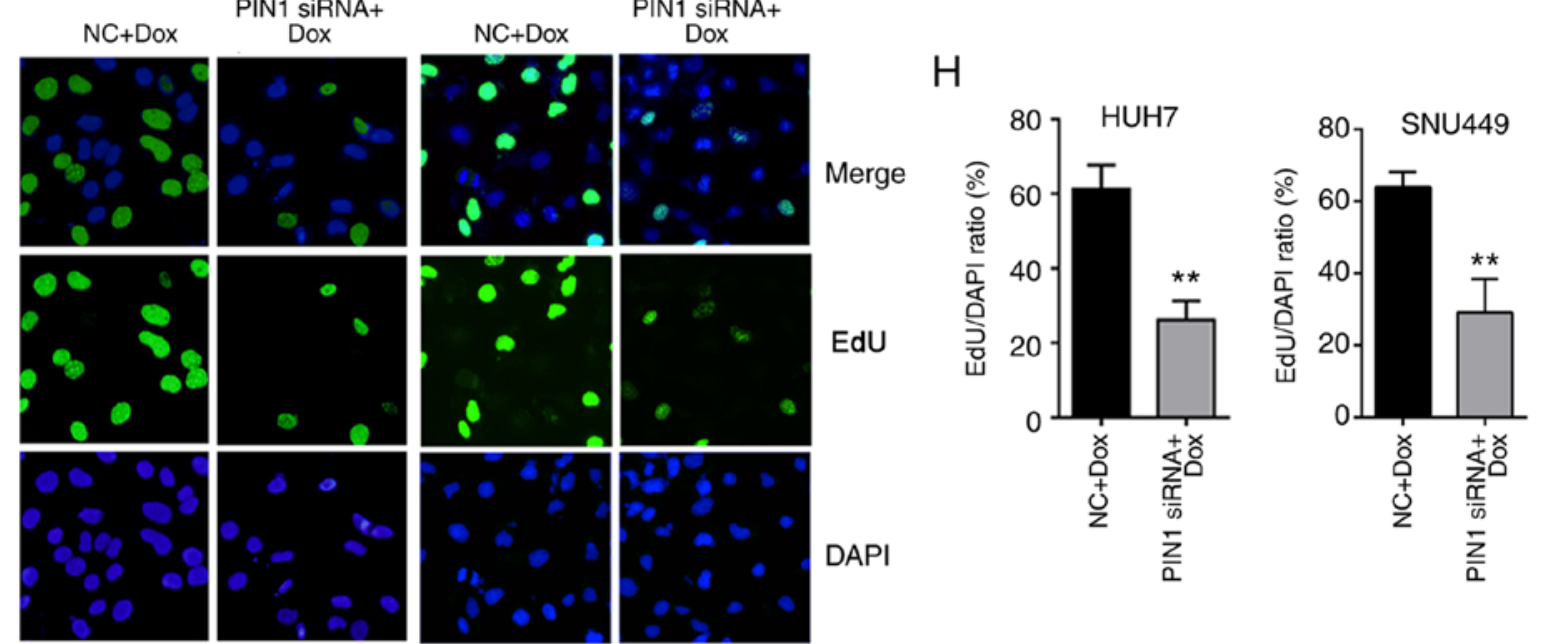

Figure 4. Knockdown of PIN1 enhances the sensitivity of HCC cells to DOX. (A) Expression level of PIN1 from starBase v3.0 database. (B) DOX treatment increased PIN1 mRNA levels in HUH7 and SNU449 cells. ${ }^{* *} \mathrm{P}<0.01$. (C) The correlation between miR-140-5p and PIN1 expression was determined by Pearson correlation analysis. (D) PIN1 protein level was decreased by transfection of PIN1 siRNA. (E and F) Silencing of PIN1 by transfection of PIN1 siRNA sensitized HUH7 and SNU449 cells towards DOX. ${ }^{* *} \mathrm{P}<0.01$. ( $\mathrm{G}$ and $\left.\mathrm{H}\right)$ Cell proliferation was measured by the EdU assay and the EdU-positive cell ratio was calculated in cells transfected with PIN1 siRNA following DOX exposure. ${ }^{* *} \mathrm{P}<0.01$. PIN1, peptidyl-prolyl cis-trans isomerase NIMA-interacting 1; HCC, hepatocellular carcinoma; DOX, doxorubicin; siRNA, small interfering RNA; NC, negative control.

and proliferation was examined using siRNA to knock down the gene in HUH7 and SNU449 cells. The PIN1 protein expression was inhibited in HUH7 and SNU449 cells transfected with PIN siRNA (Fig. 4D). HUH7 and SNU449 cells in which
PIN1 was knocked down exhibited significantly suppressed cell viability in a dose-dependent manner compared with cells transfected with NC siRNA (Fig. 4E and F). Consistently, PIN1 siRNA significantly inhibited cell proliferation in the 

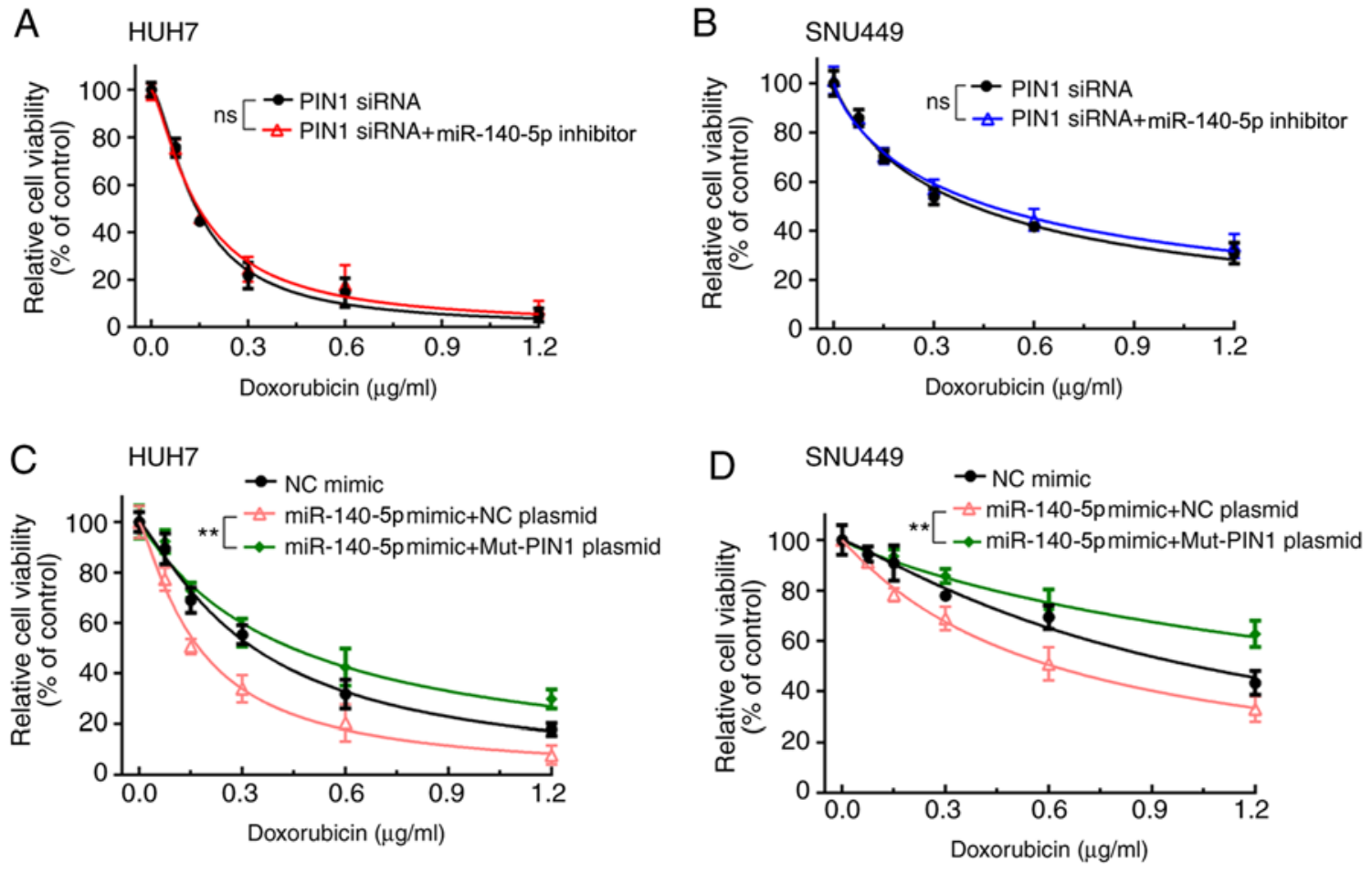

Figure 5. miR-140-5p enhances the sensitivity of HCC cells to DOX through inhibition of PIN1. (A and B) Cells were induced with PIN1 siRNA or PIN siRNA + miR-140-5p inhibitor, treated with different concentrations of DOX for $48 \mathrm{~h}$, and then cell viability was detected using the CCK- 8 assay. There was no difference in cell viability between the two groups in HUH7 and SNU449 cells. (C and D) HUH7 and SNU449 cells were induced with PIN1mut plasmid or PIN1mut plasmid + miR-140-5p mimic, treated with different concentrations of DOX for $48 \mathrm{~h}$, and then cell viability was detected using the CCK-8 assay. miR-140-5p mimic did not reduce the sensitivity of PIN1mut plasmid to DOX in HUH7 and SNU449 cells. ** $\mathrm{P}<0.01$. miR-140-5p, microRNA-140-5p; HCC, hepatocellular carcinoma; DOX, doxorubicin; PIN1, peptidyl-prolyl cis-trans isomerase NIMA-interacting 1; mut, mutant; CCK-8, Cell Counting Kit-8; NC, negative control.

presence of DOX compared with NC siRNA (Fig. 4G and H). These results demonstrated that knockdown of PIN1 increased the sensitivity of HCC cells to DOX.

miR-140-5p enhances the sensitivity of HCC cells to DOX through inhibition of PIN1. To investigate whether miR140-5p enhances the sensitivity of HCC cells to DOX by suppressing PIN1, HUH7 and SNU449 cells were transfected with PIN1 siRNA or a miR-140-5p inhibitor and PIN1 siRNA and then treated with different concentrations of DOX. The results of the CCK-8 assay revealed no significant difference in HUH7 and SNU449 cells between the two treatment groups, indicating that miR-140-5p mediated the sensitivity of HCC cells to DOX through inhibition of PIN1 (Fig. 5A and B). Furthermore, the effect of miR-140-5p mimic on the sensitivity of PIN1mut plasmid to DOX was determined in HUH7 and SNU449, revealing that miR-140-5p mimic did not reduce the sensitivity of PIN1mut plasmid to DOX in HUH7 and SNU449 cells (Fig. 5C and D).

\section{Discussion}

Recently it has been reported that miR-140-5p was significantly decreased in HCC tissues as compared with that of adjacent non-tumorous liver tissues and it suppresses tumor growth and metastasis (15). We therefore hypothesized that miR-140-5p is involved in drug resistance in HCC. In the present study, it was observed that miR-140-5p was downregulated in HCC tissues and cell lines using starBase 3.0. Of the cell lines identified in
starBase, HUH7 and SNU449 cells were selected to perform subsequent experiments. The present study revealed that miR140-5p acted as a suppressor gene in HCC. DOX is widely used for the treatment in cancers. However, resistance among cancer cells has emerged as a major barrier to effective treatment using DOX $(17,18)$. In the present study it was revealed that DOX treatment decreased miR-140-5p expression in HUH7 and SNU449 cells. The expression of miR-140-5p was higher in HUH7 cells than SNU449 after treatment with DOX IC50, while the HCC cell line HUH7 was more sensitive than SNU449 cells after treatment with DOX. The expression of miR-140-5p was higher, indicating that the sensitivity to DOX was enhanced. Subsequently, gain-of-function experiments revealed that miR140-5p mimics could enhance DOX sensitivity and decrease the proliferation rate in $\mathrm{HUH7}$ and SNU449 cells.

MicroRNAs have been demonstrated to play an important role in tumor proliferation, migration, and drug resistance (19-21). They function mainly by silencing target gene expression through imperfect base pairing with cognate transcripts. Based on this incomplete pairing, one miRNA can target multiple different mRNAs (22). TargetScan was used to identify potential target genes of miR-140-5p. Not surprisingly, 434 target genes predicted to bind to miR-140-5p were identified. Among the various target genes, PIN1 was selected to study its association with miR-140-5p.

PIN1, a highly conserved and specific polypeptide proline cis-trans isomerase, can specifically catalyze the occurrence of cis-trans isomerization of phosphorylated serine/threonineproline (pS/T-P) sequences, which affects the function of 
substrate proteins (23-25). PIN1 is a key regulator of multiple cell processes, including cell cycle progression, cell proliferation and apoptosis (26). Increasing evidence has revealed that PIN1 is aberrantly increased in most human cancers, including colorectal cancer, esophageal squamous-cell carcinoma, glioblastoma and prostate, breast and lung cancers $(25,27-29)$. In addition, in vivo, PIN1 was revealed to enhance tumorigenesis in a Li-Fraumeni mouse model (30). Yan et al (31) had demonstrated that miR-140-5p could inhibit HCC growth, migration and invasion by targeting PIN1. These findings suggested that PIN1 is a potential therapeutic target for cancer treatment. In the present study, starBase was used to observe the expression of PIN1 in HCC. Consistent with previous results, PIN1 was revealed to be higher in HCC tissues than that in the normal control tissues. In addition, PIN1 expression was detected in HUH7 and SNU449 cells following DOX exposure. It was revealed that PIN1 expression was increased in treated cells compared to the untreated cells. Based on these results, it was hypothesized that miR-140-5p may regulate DOX sensitivity via targeting PIN1.

In order to assess the correlation between miR-140-5p and PIN1, a luciferase assay was first used to investigate whether miR-140-5p could regulate the expression of PIN1 in HCC cells. It was observed that miR-140-5p mimics could significantly decrease the luciferase activity in the PIN1 3'-UTR wild-type group while no change was observed in the PIN1 3'-UTR mutation group. Furthermore, it was revealed that miR-140-5p mimics could decrease the expression of PIN1 in HUH7 and SNU449 cells. To further demonstrate this result, siRNA was used to suppress the expression of PIN1 in HUH7 and SNU449 cells. It was observed that the PIN1 siRNA efficiently knocked down PIN1 expression and this enhanced DOX sensitivity and suppressed cell proliferation in the presence of DOX in HUH7 and SNU449 cells. However, when a miR-140-5p inhibitor was co-transfected with PIN1 siRNA the DOX sensitivity was not significantly different from the PIN1 siRNA group in both the HUH7 and SNU449 cells; while miR-140-5p mimic did not reduce the sensitivity of PIN1mut plasmid to DOX in HUH7 and SNU449 cells. Thus, it was demonstrated that miR-140-5p could increase DOX sensitivity of HCC cells via targeting PIN1. However, the results of the present study were limited as the effect of miR-140-5p on DOX sensitivity was not confirmed in vivo. This should be evaluated in future studies.

In conclusion, the present study demonstrated that overexpressed miR-140-5p could enhance DOX sensitivity of HCC cells by targeting PIN1. The miR-140-5p/PIN1 axis may be a novel target for HCC targeted therapy, and further in vivo and clinical investigations are required.

\section{Acknowledgments}

Not applicable.

\section{Funding}

The present study was funded by The Project of Zhejiang Traditional Chinese Medicine Science and Technology (grant no. 2021ZB136).

\section{Availability of data and materials}

The datasets used and/or analyzed during the present study are available from the corresponding author on reasonable request.

\section{Authors' contributions}

YL conceived and designed the study. XG and YJ collected, interpreted and analyzed the data. YL wrote the manuscript. YL and XG confirm the authenticity of all the raw data. All authors read and approved the final manuscript.

\section{Ethics approval and consent to participate}

Not applicable.

\section{Patient consent for publication}

Not applicable.

\section{Competing interests}

The authors declare that they have no competing interests.

\section{References}

1. Chen B, Jin S, Bai B, Li Z, Ni C and Liu Y: Knockdown of interferon-stimulated gene 15 affects the sensitivity of hepatocellular carcinoma cells to norcantharidin. Exp Ther Med 18: 3751-3758, 2019.

2. Wang SY, Chen CL, Hu YC, Chi Y, Huang YH, Su CW, Jeng WJ, Liang YJ and Wu JC: High expression of microRNA-196a is associated with progression of hepatocellular carcinoma in younger patients. Cancers (Basel) 11: 1549, 2019.

3. Bray F, Ferlay J, Soerjomataram I, Siegel RL, Torre LA and Jemal A: Global cancer statistics 2018: GLOBOCAN estimates of incidence and mortality worldwide for 36 cancers in 185 countries. CA Cancer J Clin 68: 394-424, 2018.

4. Wu F, Zhang C, Cai J, Yang F, Liang T, Yan X, Wang H, Wang $\mathrm{W}$, Chen $\mathrm{J}$ and Jiang T: Upregulation of long noncoding RNA HOXA-AS3 promotes tumor progression and predicts poor prognosis in glioma. Oncotarget 8: 53110-53123, 2017.

5. Yang B, Wang C, Xie H, Wang Y, Huang J, Rong Y, Zhang H, Kong H, Yang Y and Lu Y: MicroRNA-3163 targets ADAM-17 and enhances the sensitivity of hepatocellular carcinoma cells to molecular targeted agents. Cell Death Dis 10: 784, 2019.

6. Zhao Y, Chen J, Wei W, Qi X, Li C and Ren J: The dual-inhibitory effect of miR-338-5p on the multidrug resistance and cell growth of hepatocellular carcinoma. Signal Transduct Target Ther 3: 3, 2018.

7. Forner A, Gilabert M, Bruix J and Raoul JL: Treatment of intermediate-stage hepatocellular carcinoma. Nat Rev Clin Oncol 11: 525-535, 2014.

8. Pérez-Tomás R: Multidrug resistance: Retrospect and prospects in anti-cancer drug treatment. Curr Med Chem 13: 1859-1876, 2006.

9. Song Y, Wang F, Huang Q, Cao Y, Zhao Y and Yang C: MicroRNAs contribute to hepatocellular carcinoma. Mini Rev Med Chem 15: 459-466, 2015.

10. Fittipaldi S, Vasuri F, Bonora S, Degiovanni A, Santandrea G, Cucchetti A, Gramantieri L, Bolondi L and D'Errico A: miRNA signature of hepatocellular carcinoma vascularization: How the controls can influence the signature. Dig Dis Sci 62: 2397-2407, 2017.

11. Masood N, Basharat Z, Khan T and Yasmin A: Entangling relation of micro RNA-let7, miRNA-200 and miRNA-125 with various cancers. Pathol Oncol Res 23: 707-715, 2017.

12. Hsu HH, Kuo WW, Shih HN, Cheng SF, Yang CK, Chen MC, Tu CC, Viswanadha VP, Liao PH and Huang CY: FOXC1 regulation of miR-31-5p confers oxaliplatin resistance by targeting LATS2 in colorectal cancer. Cancers (Basel) 11: 1576, 2019. 
13. Ma C, Shi X, Guo W, Feng F and Wang G: miR-205-5p downregulation decreases gemcitabine sensitivity of breast cancer cells via ERp29 upregulation. Exp Ther Med 18: 3525-3533, 2019.

14. Wu Y,Zhu X, Shen R, Huang J, Xu X and He S: miR-182 contributes to cell adhesion-mediated drug resistance in multiple myeloma via targeting PDCD4. Pathol Res Pract 215: 152603, 2019.

15. Yang H, Fang F, Chang R and Yang L: MicroRNA-140-5p suppresses tumor growth and metastasis by targeting transforming growth factor $\beta$ receptor 1 and fibroblast growth factor 9 in hepatocellular carcinoma. Hepatology 58: 205-217, 2013.

16. Livak KJ and Schmittgen TD: Analysis of relative gene expression data using real-time quantitative PCR and the 2(-Delta Delta C(T)) Method. Methods 25: 402-408, 2001.

17. Han S, Wang L, Sun L, Wang Y, Yao B, Chen T, Liu R and Liu Q: MicroRNA-1251-5p promotes tumor growth and metastasis of hepatocellular carcinoma by targeting AKAP12. Biomed Pharmacother 122: 109754, 2020.

18. Zhang Y, Xia F, Zhang F, Cui Y, Wang Q, Liu H and Wu Y: miR-135b-5p enhances doxorubicin-sensitivity of breast cancer cells through targeting anterior gradient 2. J Exp Clin Cancer Res 38: 26, 2019.

19. Wang Y, Ao X, Liu Y, Ding D, Jiao WJ, Yu Z, Zhai WX, Dong SH, $\mathrm{He} \mathrm{YQ}$, Guo $\mathrm{H}$, et al: MicroRNA-608 promotes apoptosis in non-small cell lung cancer cells treated with doxorubicin through the inhibition of TFAP4. Front Genet 10: 809, 2019.

20. Gu N, Wang X, Di Z, Xiong J, Ma Y, Yan Y, Qian Y, Zhang Q and Yu J: Silencing lncRNA FOXD2-AS1 inhibits proliferation, migration, invasion and drug resistance of drug-resistant glioma cells and promotes their apoptosis via microRNA-98-5p/CPEB4 axis. Aging (Albany NY) 11: 10266-10283, 2019.

21. Patil SL, Palat A, Pan Y, Rajapakshe K, Mirchandani R, Bondesson M, Yustein JT, Coarfa C and Gunaratne PH: MicroRNA-509-3p inhibits cellular migration, invasion, and proliferation, and sensitizes osteosarcoma to cisplatin. Sci Rep 9: 19089, 2019

22. Lim LP, Lau NC, Garrett-Engele P, Grimson A, Schelter JM, Castle J, Bartel DP, Linsley PS and Johnson JM: Microarray analysis shows that some microRNAs downregulate large numbers of target mRNAs. Nature 433: 769-773, 2005.
23. Campaner E, Rustighi A, Zannini A, Cristiani A, Piazza S, Ciani Y, Kalid O, Golan G, Baloglu E, Shacham S, et al: A covalent PIN1 inhibitor selectively targets cancer cells by a dual mechanism of action. Nat Commun 8: 15772, 2017.

24. Zheng Y, Pu W, Li J, Shen X, Zhou Q, Fan X, Yang SY, Yu Y, Chen $\mathrm{Q}$, Wang $\mathrm{C}$, et al: Discovery of a prenylated flavonol derivative as a Pin1 inhibitor to suppress hepatocellular carcinoma by modulating microRNA biogenesis. Chem Asian J 14: 130-134, 2019.

25. Lu Z and Hunter T: Prolyl isomerase Pin1 in cancer. Cell Res 24: 1033-1049, 2014

26. Yeh ES and Means AR: PIN1, the cell cycle and cancer. Nat Rev Cancer 7: 381-388, 2007.

27. Atkinson GP, Nozell SE, Harrison DK, Stonecypher MS, Chen D and Benveniste EN: The prolyl isomerase Pin1 regulates the NF-kappaB signaling pathway and interleukin-8 expression in glioblastoma. Oncogene 28: 3735-3745, 2009.

28. Pyo JS, Son BK and Oh IH: Cytoplasmic Pin1 expression is correlated with poor prognosis in colorectal cancer. Pathol Res Pract 214: 1848-1853, 2018.

29. Chen M, Xia Y, Tan Y, Jiang G, Jin H and Chen Y: Downregulation of microRNA-370 in esophageal squamous-cell carcinoma is associated with cancer progression and promotes cancer cell proliferation via upregulating PIN1. Gene 661: 68-77, 2018.

30. Girardini JE, Napoli M, Piazza S, Rustighi A, Marotta C, Radaelli E, Capaci V, Jordan L, Quinlan P, Thompson A, et al: A Pin 1/mutant p53 axis promotes aggressiveness in breast cancer. Cancer Cell 20: 79-91, 2011.

31. Yan X, Zhu Z, Xu S, Yang LN, Liao XH, Zheng M, Yang D, Wang J, Chen D, Wang L, et al: MicroRNA-140-5p inhibits hepatocellular carcinoma by directly targeting the unique isomerase Pin1 to block multiple cancer-driving pathways. Sci Rep 7: 45915, 2017.

(i) (5) $\odot$ This work is licensed under a Creative Commons Attribution-NonCommercial-NoDerivatives 4.0 International (CC BY-NC-ND 4.0) License. 